\title{
Metis: a profiling toolkit based on the virtualization of hardware performance counters
}

\author{
Xia Xie ${ }^{1 *}$, Haiou Jiang ${ }^{1}$, Hai Jin ${ }^{1}$, Wenzhi Cao ${ }^{1}$, Pingpeng Yuan ${ }^{1}$ and Laurence Tianruo Yang ${ }^{2}$
}

\author{
* Correspondence: shelicy@hust. \\ edu.cn \\ 'Services Computing Technology \\ and System Lab, Cluster and Grid \\ Computing Lab, School of \\ Computer Science and \\ Technology, Huazhong University \\ of Science and Technology, Wuhan \\ 430074, China \\ Full list of author information is \\ available at the end of the article
}

\begin{abstract}
Background: With wide application of virtualization technology, the demand is increasing for performance analysis and system diagnosis in virtualization environment. There are some profiling toolkits based on hardware events, such as OProfile in native Linux and Xenoprof in Xen virtual machine environment. However, sometimes users in different domains need monitor different hardware events individually at the same time. For programming and profiling in environment for virtual machine, it may become popular in the coming future. In this paper, we present Metis, a system-wide profiling toolkit for Xen virtual machine environment based on the virtualization of hardware performance counters.

Methods: Virtualization of hardware performance counters is used to enable profiling of processes and routines running in the domain or Xen virtual machine monitor.

Results: This toolkit allows multiple users in different domains to monitor different hardware events simultaneously in Xen virtual machine environment, obtaining the distribution of hardware events such as clock cycles, instruction execution and cache misses, etc. Our experiments with a real-world benchmark demonstrate the good performance of Metis.
\end{abstract}

Conclusion: Comparing to all the existing profiling toolkits, Metis is different which enables multiple users in different virtual machines to monitor different CPU events simultaneously, and users in different domains can use this toolkit individually without affecting each other. We apply a popular benchmark to verify the correctness of Metis and its cool features.

Keywords: Hardware Performance Counter, CPU Event, Virtual Machine, Profile

\section{Introduction}

Virtualization technology allows multiple Virtual Machines (VMs) running on a single physical host. Each VM can run different Operating System (OS), applications in respective OS can run individually without affecting each other. Many enterprise applications traditionally running on dedicated servers consolidate to a smaller and shared pool of servers [1]. Xen is an x86 virtual machine monitor, which allows multiple commodity operating systems to share conventional hardware in a safe and resource managed fashion without sacrificing either performance or functionality [2]. Even some cases for high performance computing with virtual machines can achieve almost the same performance as those running in a native, non-virtualized environment $[3,4]$.

(c) 2012 Xie et al; licensee Springer. This is an Open Access article distributed under the terms of the Creative Commons Attribution License (http://creativecommons.org/licenses/by/2.0), which permits unrestricted use, distribution, and reproduction in any medium, provided the original work is properly cited. 
With the wide application of virtualization technology, the demand is increasing for performance analysis and system diagnosis in virtualization environment [5].

Xenoprof is a system-wide statistical profiling toolkit, which is modeled on the OProfile profiling toolkit and available for Linux systems. The Xenoprof toolkit supports system-wide coordinated profiling in a Xen environment to obtain the distribution of hardware events, such as clock cycles, instruction execution, TLB and cache misses. However, since domains (Xen uses the term "domain" to refer to a virtual machine) are in a coordinated profiling session when Xenoprof is used, they should have the same hardware events configuration and obtain respective performance data. Sometimes, users in different domains may have the demand to monitor different hardware events simultaneously. For example, a user is in A domain, and want to monitor two events, such as CPU_CLK_UNHALTED and ITLB_MISS_RETIRED, while another user logs in B domain and need to use another two hardware events such as DTLB_MISSES and BUS_TRAN_MEM at the same time. A new profiling toolkit is preferred to make multiple users monitor different events individually at any time without affecting each other.

In this paper, we present Metis, a system-wide profiling toolkit for Xen virtual machine environment. It is depended on the virtualization of hardware performance counters. This toolkit enables profiling of processes and routines running in domain or Xen virtual machine monitor (VMM, which is intrigued both researchers and entrepreneurs [6]). It allows multiple users in different domains to monitor different hardware events simultaneously in Xen virtual machine environment, for obtaining the distribution of hardware events such as clock cycles, instruction execution, TLB and cache misses, etc. Though the number of hardware performance counters is limited, the number of hardware events that a domain can monitor is also dependent on it because of the virtualization of hardware performance counters.

We present an experiment in a physical host with two hardware performance counters, and monitor two events (CPU_CLK_UNHALTED and ITLB_MISS_RETIRED) in a domain, while another two events (DTLB_MISSES and BUS_TRAN_MEM) are monitored in another domain. It shows the effect of the virtualization of hardware performance counters in detail. Besides this, we report on the use of Metis to analyze performance overheads for Gzip (a benchmark in SPEC CPU2000) by monitoring two hardware events such as CPU_CLK_UNHALTED and ITLB_MISS_RETIRED. Two users in two different domains start and stop Metis simultaneously in the experiment, and we compare the performance data with the output produced by Xenoprof.

The rest sections are organized as follows. Section 2 discusses the related works about profiling toolkits. Section 3 details the design and implementation of Metis on Xen. In Section 4, we test Metis with popular benchmark and analyze the experiments. We draw the conclusion in section 5 .

\section{Related works}

There are a few profiling toolkits which use hardware performance counters, such as OProfile, VTune in native Linux and Xenoprof in Xen virtual machine environment. In this section, we briefly describe these toolkits, and then we present our motivation for this work. 


\section{Oprofile}

OProfile is a system-wide statistical profiling tool for Linux systems. We can use OProfile to profile code executing at any privilege level, including kernel code, kernel modules, user level applications and user level libraries.

OProfile uses performance-monitoring hardware on processors to collect periodic samples of various performance data. Performance monitoring hardware on modern processor architectures include counters that track various processor events including clock cycles, instruction retirements [7], TLB misses, cache misses, branch mis-predictions, etc. Researchers can configure the performance-monitoring hardware to notify the operating system when these counters reach specified values. Profiles are generated by regularly sampling the current registers on each CPU and converting the runtime $\mathrm{PC}$ value into something meaningful to the programmer. OProfile achieves this by taking the stream of sampled PC values, along with the detail which task was running at the time of the interrupt, and converting it into a file offset against a particular binary file. Because applications map the code that they run by using mmap(), it's possible to find the relevant binary file and offset by walking the task's list of mapped memory areas. Then the user-space tools can reconstruct where the code came from, including the particular assembly instructions, symbol, and source line (via the binary's debug information if present).

Profiling with OProfile operates as follows [8]:

1. User provides input for OProfile about the performance events to be monitored and the periodic count.

2. OProfile programs hardware counters to calculate the number of user-specified performance events and to generate a non-maskable interrupt (NMI) when the counter has counted to the user-specified count.

3. The performance-monitoring hardware generates an NMI according to counter overflow.

4. OProfile's NMI handler catches the NMI and records the program counter value in a kernel buffer.

5. OProfile processes the buffer periodically to determine the routine and executable corresponding to the program counter on each sample in the buffer. This is determined by consulting the virtual memory layout of the process and the kernel.

\section{VTune}

Intel VTune Performance analyzer is a commercial application for software performance analysis about Intel-manufactured x86 and x64 based machines, and has both GUI and command line interfaces. It is available for both Linux and Microsoft Windows operating systems.

Intel VTune Performance analyzer assists in various kinds of code profiling including time-based profiling, event-based profiling. The profiler result consists of details such as time spent in each sub routine, which is drilled down to the instruction level. The time taken by the instructions is indicative of any stalls in the pipeline during instruction execution. The tool can be also used to analyze thread performance $[9,10]$. 


\section{Xenoprof}

Xenoprof is a system-wide profiler for Xen virtual machine environments, capable of profiling the Xen VMM, multiple Linux guest operating systems, and applications running on them [11].

The Xenoprof toolkit provides capabilities similar with OProfile for the Xen environment (i.e., using performance-monitoring hardware to collect periodic samples of performance data). The performance of applications running on Xen depends on interactions among the application's processes, the operating system it is running on, the Xen VMM, and potentially other virtual machines (e.g., driver domain) running on the same system. In order to study the costs of virtualization and the interactions among multiple domains, the performance profiling tool must be able to determine the distribution of performance events across routines in the Xen VMM and all the domains running on it.

In virtual machine environments like Xen, a major source of performance degradation is the cost of $\mathrm{I} / \mathrm{O}$ devices virtualization to allow multiple guest VMs to share a single device securely [12], and we can use Xenoprof to measure the I/O performance meanwhile. We also use information extracted by Xenoprof to uncover bugs and channel the information into optimizing Xen, to provide a view of their application on virtual machines for a scientist or an application's user [13]. In [14], researchers use Xenoprof for memory intensive applications such as DGEMM.

The Xenoprof toolkit consists of a VMM-level layer responsible for servicing counter overflow interrupts from the performance monitoring hardware and a domain-level layer derived from OProfile responsible for attributing samples to specific routines within the domain [15]. The OProfile layer drives the performance profiling through hypercalls supported by Xenoprof and Xenoprof delivers samples to the OProfile layer using Xen's virtual interrupt mechanism. System-wide profiling is generated through the coordination of multiple domain-level profilers. While our current implementation is dependent on OProfile, other statistical profilers (e.g., VTune) also can be ported for using the Xenoprof interface.

Domain level profilers must be ported to the Xenoprof interface so that they can be used in the Xen environment. Porting a profiler to Xenoprof is very straightforward, and entails the following steps:

1. We modify the profiler code for accessing and programming the hardware counters by using the Xenoprof virtual event interface.

2. Before starting profiling, the profiler queries Xenoprof to determine whether it is to take on the role of the initiator. Only the initiator domain performs the global profiling setup, such as deciding the events and profiling duration. Both the initiator and the other profilers register their callback functions for collecting PC samples.

3. We extend the profiler with access to the Xen virtual memory layout, so that it can identify Xen routines corresponding to PC samples in Xen's virtual address range.

We put forward a system-wide profiling toolkit for Xen virtual machine environment, which is dependent on the virtualization of hardware performance counters. This toolkit enables multiple users in different domains to profile different hardware events simultaneously in Xen virtual machine environment, without affecting each other. 


\section{System Design and Implementation}

\section{System framework and overview}

The toolkit consists of two parts: kernel level and user level. Key processing are mainly concentrated in the kernel level (including Xen and Linux), such as the configuration and virtualization of hardware performance counters, sampling mechanism and etc., while use-space daemons of virtual machines are primarily used to analyze samples from the buffer in the kernel which are connected with corresponding functions or sections. Finally, the performance analysis data is shown for users clearly. The framework of this toolkit is as follows in Figure 1.

Figure 2 shows the workflow of Metis, and the whole process schedule of workflow is as follows:

Step 1 A background daemon is running in each privileged domain or guest domain to monitor the operations triggered by users, such as starting or stopping profiling.

Step 2 The user in respective privileged domain or guest domain sets the CPU events to profile. If the count of CPU events in a domain is bigger than the number of hardware performance counters, the user will be alerted that he should reduce the number of CPU events, and then repeat this step.

Step 3 The user starts profiling, and send a starting signal to the privileged domain. Then the CPU events configuration is written to respective structures of Virtual CPU (VCPU):

Step 3.1 All CPU events configurations of the domains are stored in the monitor. (Each VCPU belongs to a domain, and each domain has respective CPU events configuration, including $\mathrm{CPU}$ event name, sampling frequency, etc.)

Step 3.2 After the user in a domain sets CPU events to monitor and starts profiling, add corresponding CPU event item for this domain in respective structures of VCPU.

Step 4 If the total count of all CPU events is bigger than the number of hardware performance counters in the machine, the virtualization of hardware performance counters is used, and go to step 5. Otherwise, go to step 6 directly.

Step 5Start the virtualization of hardware performance counters (time-sharing multiplexing technology).

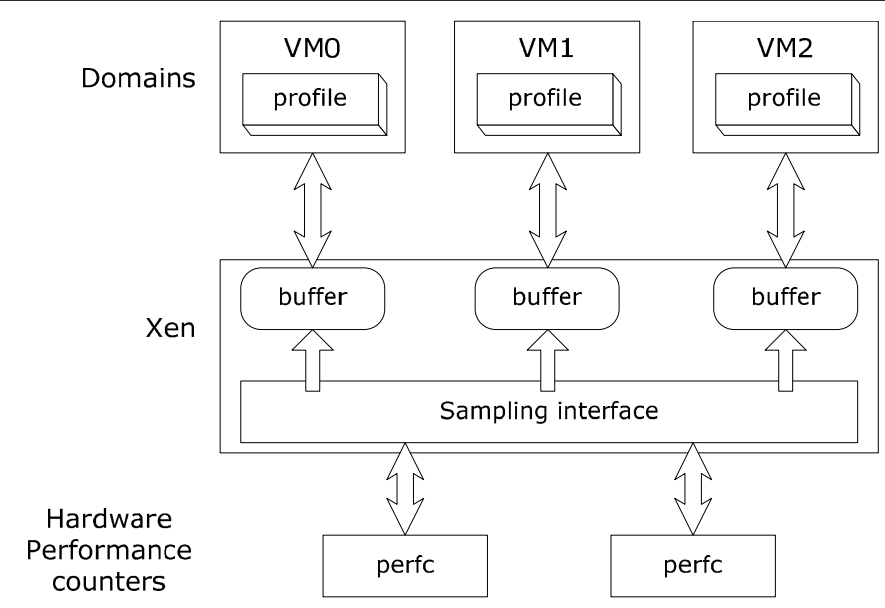

Figure 1 Metis's Framework. 


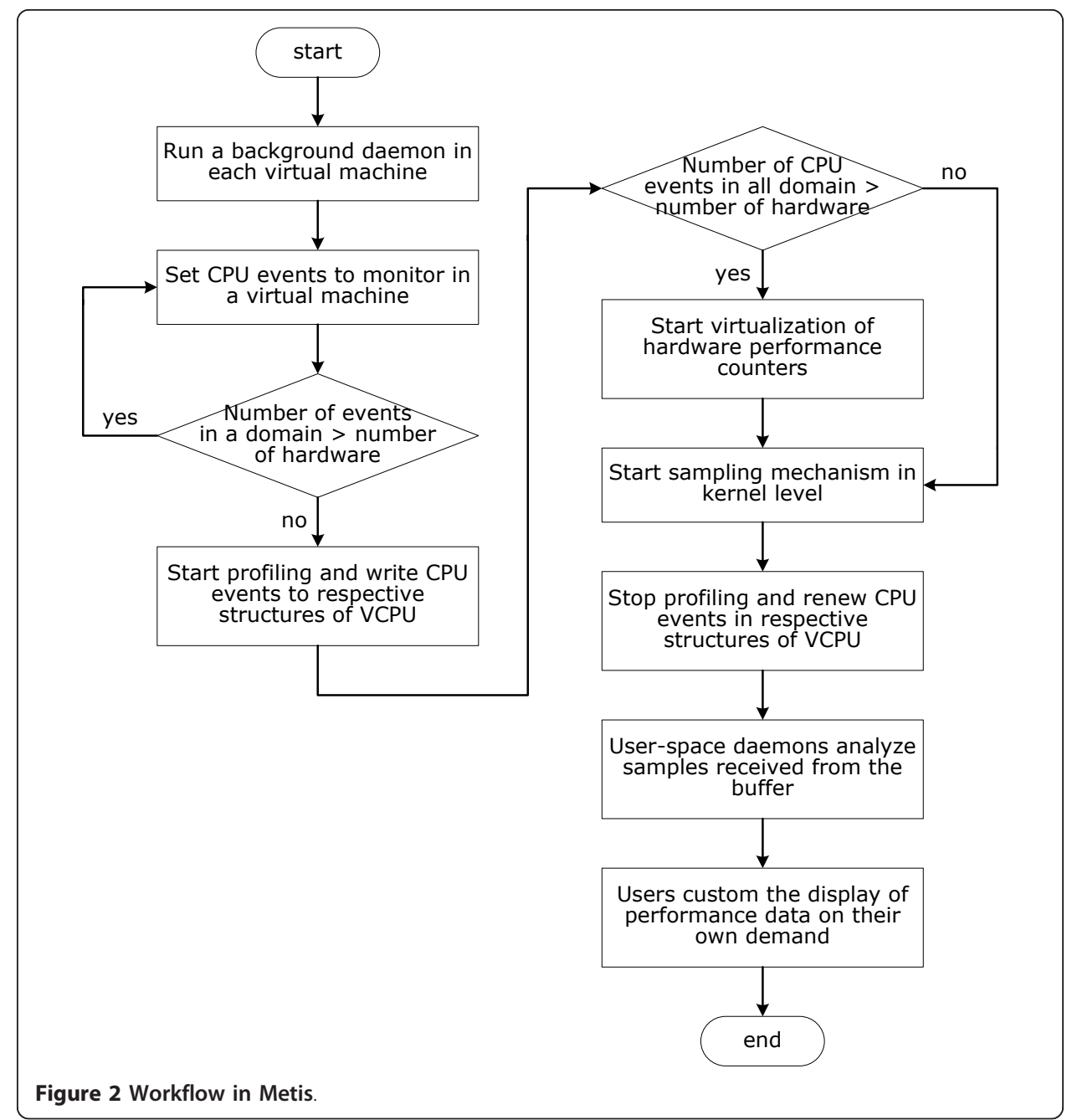

Step 6 Start sampling mechanism in kernel level. When the current count of a CPU event in a hardware performance counter reaches the number set for such CPU event, an interrupt occurs to record current PC (program counter) value and other context information, and save the information into corresponding buffer, and meanwhile the system reset counter value. The sampling data is distributed to respective domain for analysis by user-space daemons later.

Step 7 When profiling stops in a domain, a stopping signal is sent to privileged domain, and corresponding CPU event item is removed from respective structures of VCPU.

Step 8 The user-space daemons are in charge of the analysis of samples received from the buffer, and after this processing we can get the hotspot, even the source code line (which causes the hotspot) will be obtained if debug information exists.

Step 9 Users can custom the display of performance data on their own demand, such as function calling diagram, the hotspots (functions or modules), etc.

\section{Read and write hardware performance counters}

In computers, hardware performance counters, or hardware counters are a set of special-purpose registers built into modern microprocessors to store the counts of 
hardware-related activities within computer systems. Advanced users often rely on those counters to conduct low-level performance analysis or optimizing.

The number of available hardware counters in a processor is limited while each CPU model might have many different events that a developer might like to measure. Each counter can be programmed with the index of an event type to be monitored, like a L1 cache miss or a branch misprediction [16].

Regard Intel Itanium 2 performance monitoring unit as an example, it organizes the counters as four pairs of performance event monitoring registers. Each pair is composed of a Performance Monitoring Configuration (PMC) register and Performance Monitoring Data (PMD) register. The PMC selects the performance event monitored and the PMD determines the sampling interval [17].

When a CPU event is monitored, you should configure the attributes of this event and then set PMC register and PMD register. All attributes of a CPU event are as follows Table 1:

For example, in user space, when we want to set the PMC register, we can call perfmonctl with the parameter cmd valued PFM_WRITE_PMCS, while the parameter $\mathrm{cm}$. will be set to PFM_WRITE_PMDS if we need to set the PMD register.

In Xen, functions such as xenpfm_write_pmcs or xenpfm_write_pmds will be called to set the hardware performance counters.

\section{Message delivery mechanism in domains}

The privileged virtual machine is called Dom0, while a guest virtual machine is called DomU. We run specific daemons in Dom0 and DomU, in which place users are willing to use this profiling toolkit, and use Xenstore as the message delivery mechanism in domains.

Xenstore is a database for Xen applications and drivers to communicate and store configuration information. Applications and tools should use the store to configure drivers by writing information into keys in the database; drivers should set watches on the appropriate keys and respond to changes appropriately [18]. Xenstore is an information storage space shared between domains. It is meant for configuration and status information rather than for large data transfers. Each domain gets its own path in the store, which is somewhat similar in spirit to procfs. When values change in the store, the appropriate drivers are notified [19].

The process how this toolkit handles in Dom0 and DomU is different. Details are shown in the following:

1. When Dom0 starts profiling, it will use Xenstore to send a signal. As soon as the background daemon in Dom0 catches the signal, it will initialize the beginning processes and start relative daemons. Finally, we clear relative information in Xenstore. When Dom0 stops profiling, the process is similar to starting (shown as Figure 3).

Table 1 All attributes of a CPU event

\begin{tabular}{ll}
\hline Event & The event type e.g. CPU_CLK_UNHALTED \\
unit mask & The sub-events to count \\
counter & The hardware counter(s) that can count this event \\
Count & The reset value (how many events before an interrupt) \\
Kernel & Whether the counter should increment when in kernel space \\
User & Whether the counter should increment when in user space \\
\hline
\end{tabular}




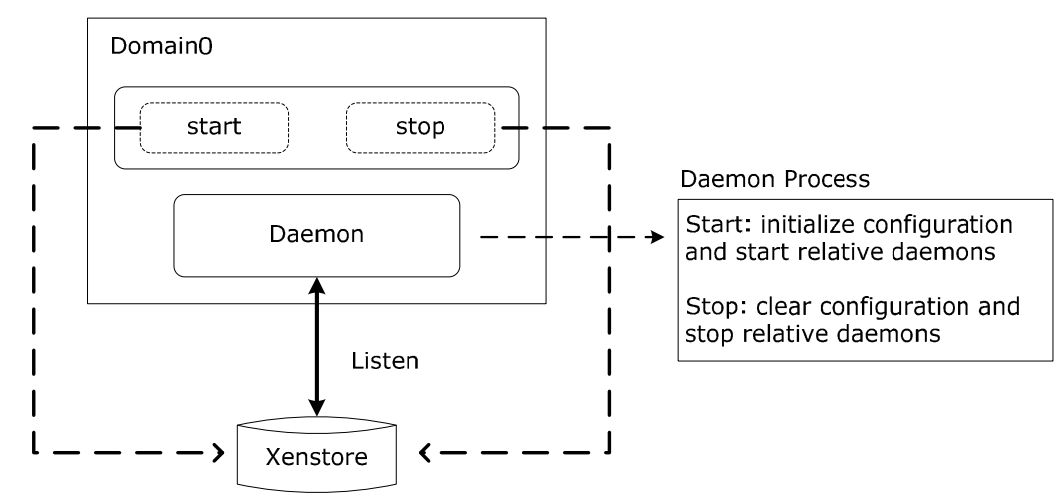

Figure 3 Start or stop profiling in Dom0

2. When DomU starts profiling, it will use Xenstore to send a signal. As soon as the background daemon in Dom0 catches the signal, it will initialize the beginning processes for DomU, and use Xenstore to notify the profiling daemon running in DomU to start relative analysis daemons and other operations. Finally, we clear relative information in Xenstore. When DomU stops profiling, the process is similar to starting (shown as Figure 4)

\section{Virtualization of hardware performance counters}

To ensure multiple virtual machines use hardware performance counters individually, we implement the virtualization of hardware performance counters (shown as Figure 5), and the detailed steps are showed below:

Step 1 when a VCPU is scheduled out while another VCPU is scheduled in, judge whether these two VCPU belong to the same virtual machine. If yes, do nothing to hardware performance counters during this VCPU schedule period, and skip step 2 and step 3. Otherwise, do step 2.

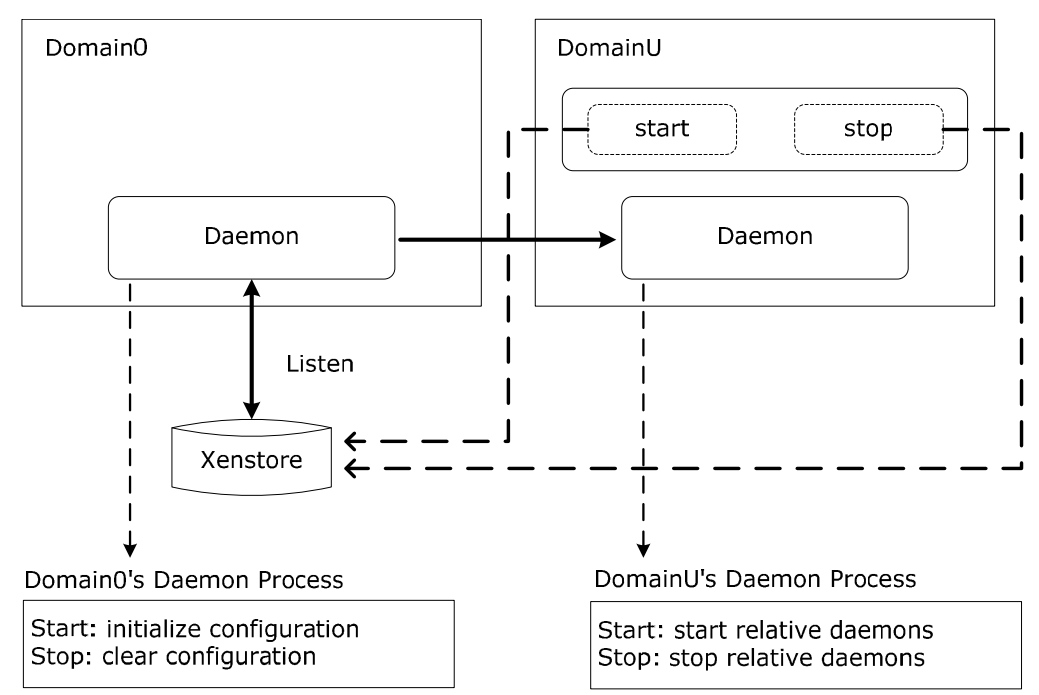

Figure 4 Start or stop profiling in DomU. 


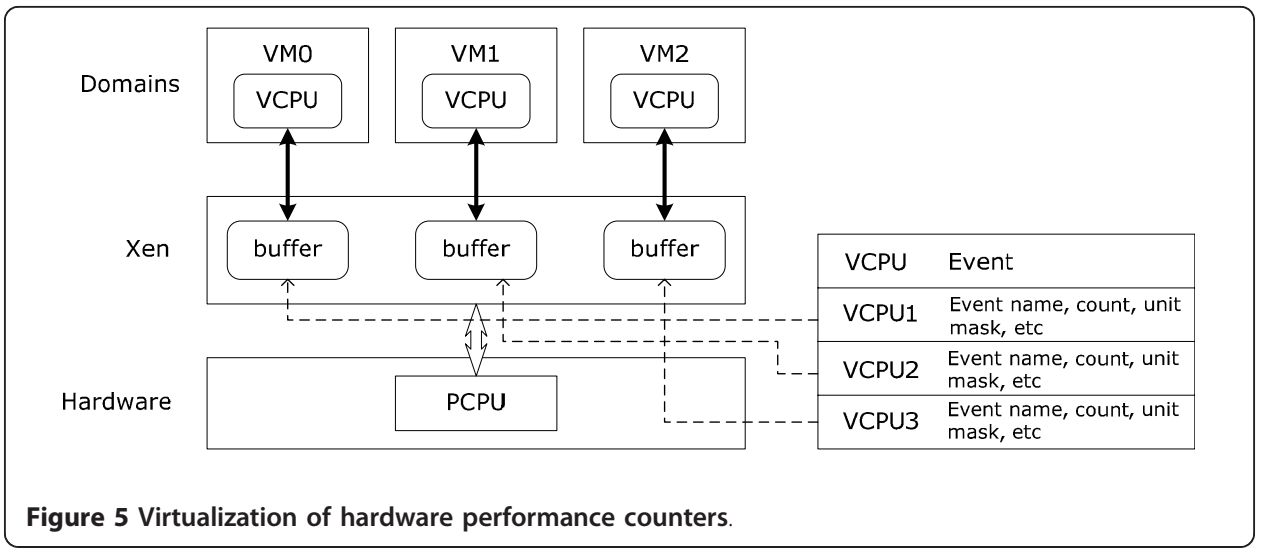

Step 2 Read the values of hardware performance counters responding to the respective CPU events for the virtual machine, which the previous VCPU belongs to, and store them for restoration when the virtual machine is scheduled to run next time.

Step 3 Read the stored counts of respective CPU events for the virtual machine, which the next VCPU belongs to, meanwhile get CPU events configured by the virtual machine, and reconfigure the hardware performance counters for CPU events monitored by the virtual machine to run.

\section{Ensure profiling accuracy}

To ensure the profiling accuracy, we put read/write operations of hardware performance counters in the VMM time slice instead of VCPU time slice, as we can see in Figure 6. During this process, we always need to restore or reset the hardware performance counters to satisfy the need of monitoring different CPU events in different domains. This operation will affect little to running domains and help reduce some performance cost in domains.

Because hardware performance counters are generally used by a certain profile toolkit instead of other processes, we ignore the samples corresponding to the processes on hardware performance counters during the analysis of samples, which can reduce the interference from hardware performance counters.

\section{Performance Evaluations}

In this section, we present an experiment on a physical host with two hardware performance counters, to monitor two events (CPU_CLK_UNHALTED and ITLB_MISS_RETIRED) in a domain, while another two events (DTLB_MISSES and

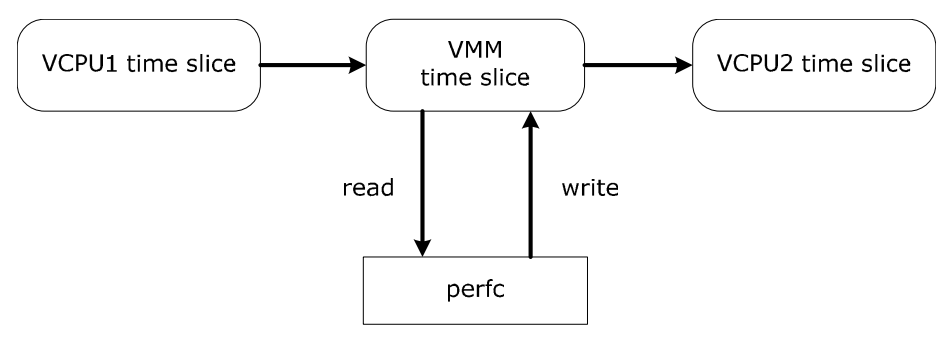

Figure 6 A way to ensure profiling accuracy. 
BUS_TRAN_MEM) are monitored in another domain. It shows the effect of the virtualization of hardware performance counters. Besides this, we report the use of Metis to analyze performance overheads of Gzip (a benchmark in SPEC CPU2000) by monitoring two hardware events such as CPU_CLK_UNHALTED and ITLB_MISS_RETIRED. Two users in two domains start and stop Metis simultaneously in the experiment, and we compare the performance data with the output produced by Xenoprof.

\section{Experimental Setup}

We built our experimental environment on a server, which has 4 Intel Xeon $1.6 \mathrm{GHz}$ CPUs, 4 GB DDR RAM and 150 GB hard disk. We used Linux 2.6.18 with Xen 3.3.1 installed as the operation system. We run Xen with two guest domains configured with equal resource allocations [20]. In all cases the VM was configured to have a single CPU with $512 \mathrm{MB}$ of RAM, and installed a Red Hat Enterprise Linux 5.3 as guest OS. To execute evaluation, we create our test environment where two domains (domain1 and domain2) run. The experiment environment is listed as Figure 7.

We present two experiments in this section. One is to prove that Metis enables two domains to monitor different CPU events simultaneously without affecting each other. Another is used to compare the performance data generated by Metis with the result produced by Xenoprof when the gzip benchmark is running.

\section{Functional experiment}

We can use this profiling toolkit individually in either domain1 or domain2, which enables domain 1 and domain2 to monitor different CPU events simultaneously. For example, we monitor CPU_CLK_UNHALTED and ITLB_MISS_RETIRED in domain1, while DTLB_MISSES and BUS_TRAN_MEM are monitored in domain2 at the same time. There are two hardware performance counters in the server, so each domain can monitor two CPU events each time without affecting each other.

We have done an experiment in two domains (and two domains are enough for indicating advantage of VirtOprof). We use domain1 to represent a priviledged domain, while domain2 is used to represent a guest domain, and more guest domains are not necessary because they share all CPU time slices and will not affect each other. We monitor CPU_CLK_UNHALTED and ITLB_MISS_RETIRED in domain1, while DTLB_MISSES and BUS_TRAN_MEM are monitored in domain2 at the same time. We start and stop Metis in two domains simultaneously, and respective performance

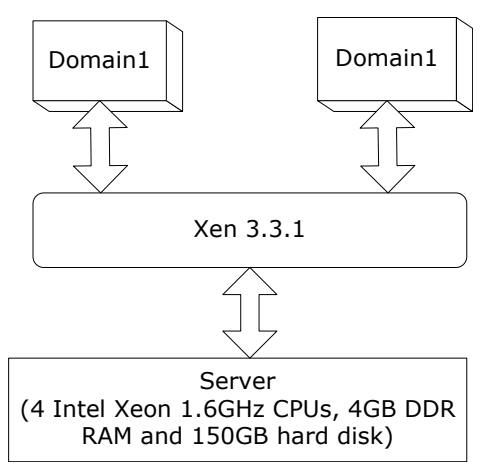

Figure 7 The experiment environment. 
data can be obtained. We can use Metis to get the performance data of each running domain.

The performance data in domain 1 are showed in Figure 8 and 9 below:

In Figure 8, we can get CPU usage rate of each symbol, and it is clear that the symbol named "xen-syms-3.3.1" and the symbol named "vmlinux-2.6.18.8-xen" consumed most of CPU resources.

In Figure 9, we can get ITLB miss rate of each symbol, and it's seen that the symbol named "vmlinux-2.6.18.8-xen" generated most of ITLB misses, while ITLB miss rate of the symbol named "xen-syms-3.3.1" and the symbol named "xen-unknown" are followed it.

In Figure 10, we can see that the symbol named "vmlinux-2.6.18.8-xen" is the main source of DTLB misses, while the symbol named "xen-syms-3.3.1" is followed. Moreover, these two symbols take up most of the whole samples.

In Figure 11, we can get BUS_TRAN_MEM sample rate of each symbol, and it is shown that the symbol named "vmlinux-2.6.18.8-xen" generated more than a half of total samples, while the symbol named "xen-syms-3.3.1" takes up about one third.

We can see that Metis allows two different domains to monitor different CPU events individually, which cann't be supported with Xenoprof, which is based on hardware performance counters in Xen environment.

\section{Performance comparison}

SPEC CPU2000 is the next-generation industry-standardized CPU-intensive benchmark suite. SPEC designed CPU2000 to provide a comparative measure of compute intensive performance across the widest practical range of hardware. The implementation resulted in source code benchmarks and developed from real user applications. These benchmarks measure the performance of the processor, memory and compiler on the tested system [15].

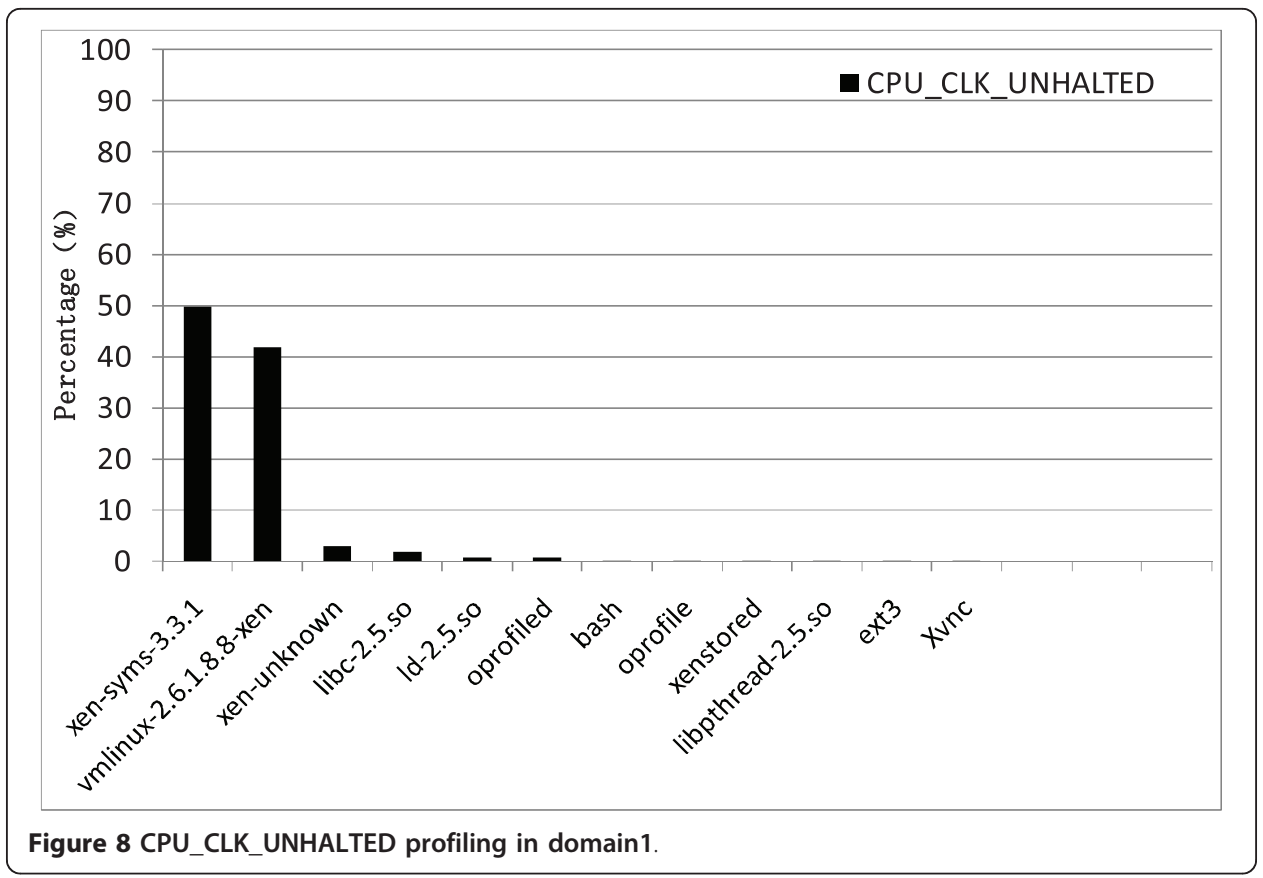




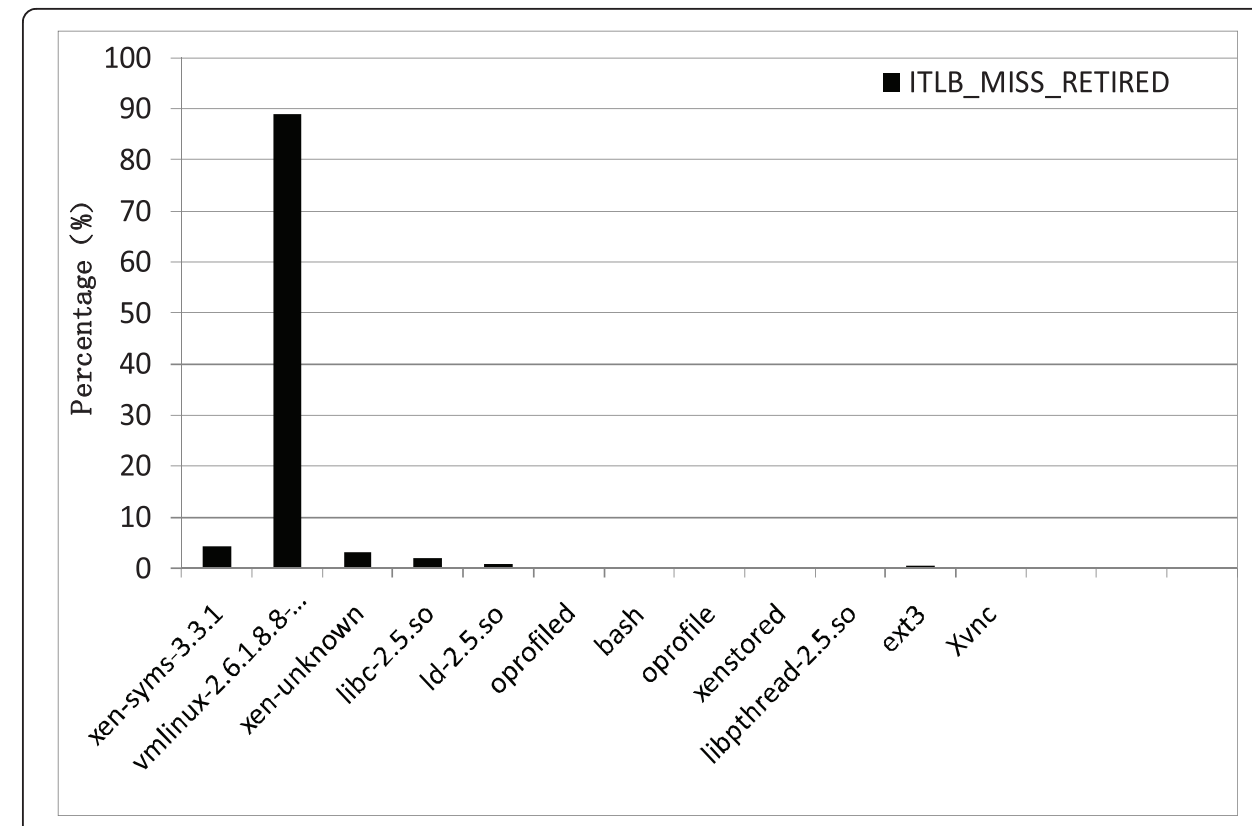

Figure 9 ITLB_MISS_RETIRED profiling in domain1.

Gzip is a benchmark in SPEC CPU2000. We choose it running in a virtual machine to compare the performance data generated by Metis with the output produced by Xenoprof. Two CPU events are monitored, such as CPU_CLK_UNHALTED and ITLB_MISS_RETIRED. We finally get the CPU usage rate and ITLB miss rate of each function in Gzip.

We compare two outputs generated by Metis and Xenoprof, and draw histograms where we can clearly get hotspots in respective CPU event (CPU_CLK_UNHALTED and ITLB_MISS_RETIRED). These two histograms are followed:

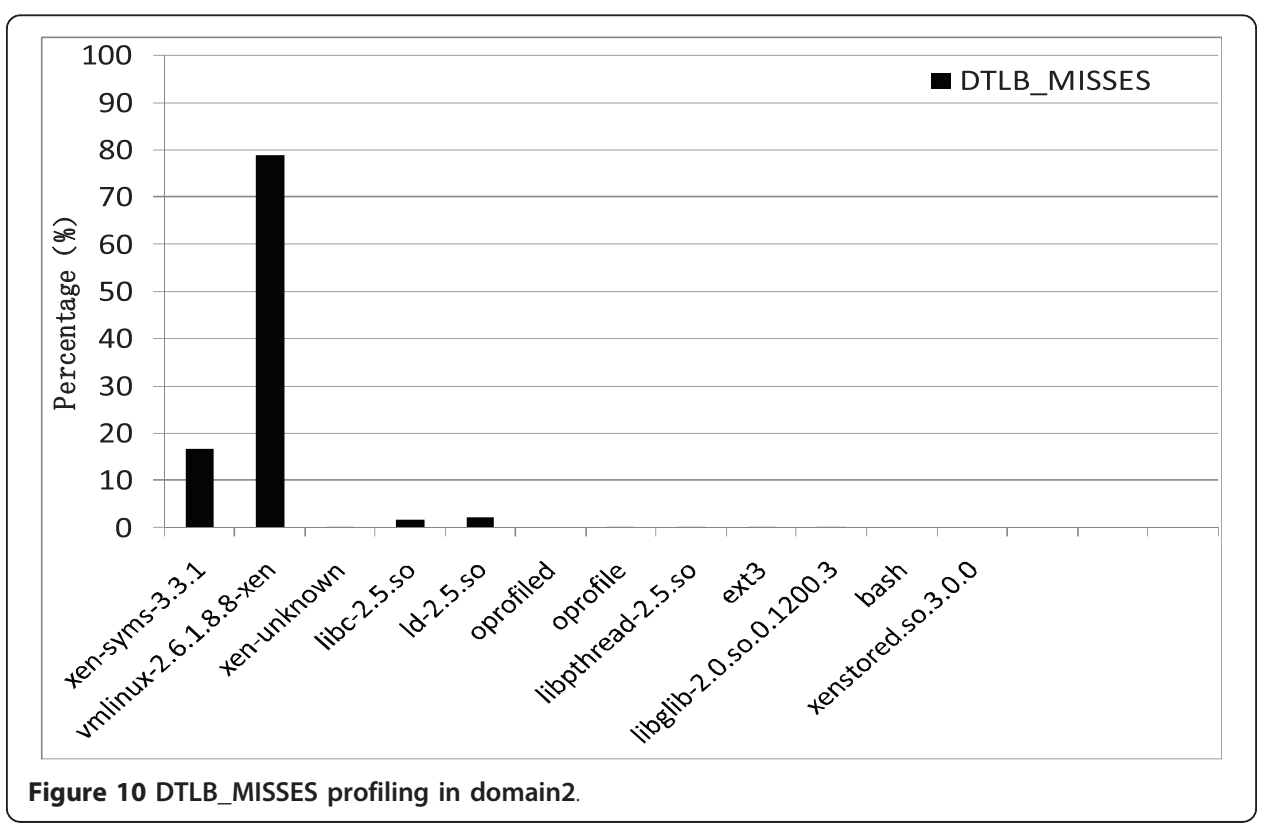




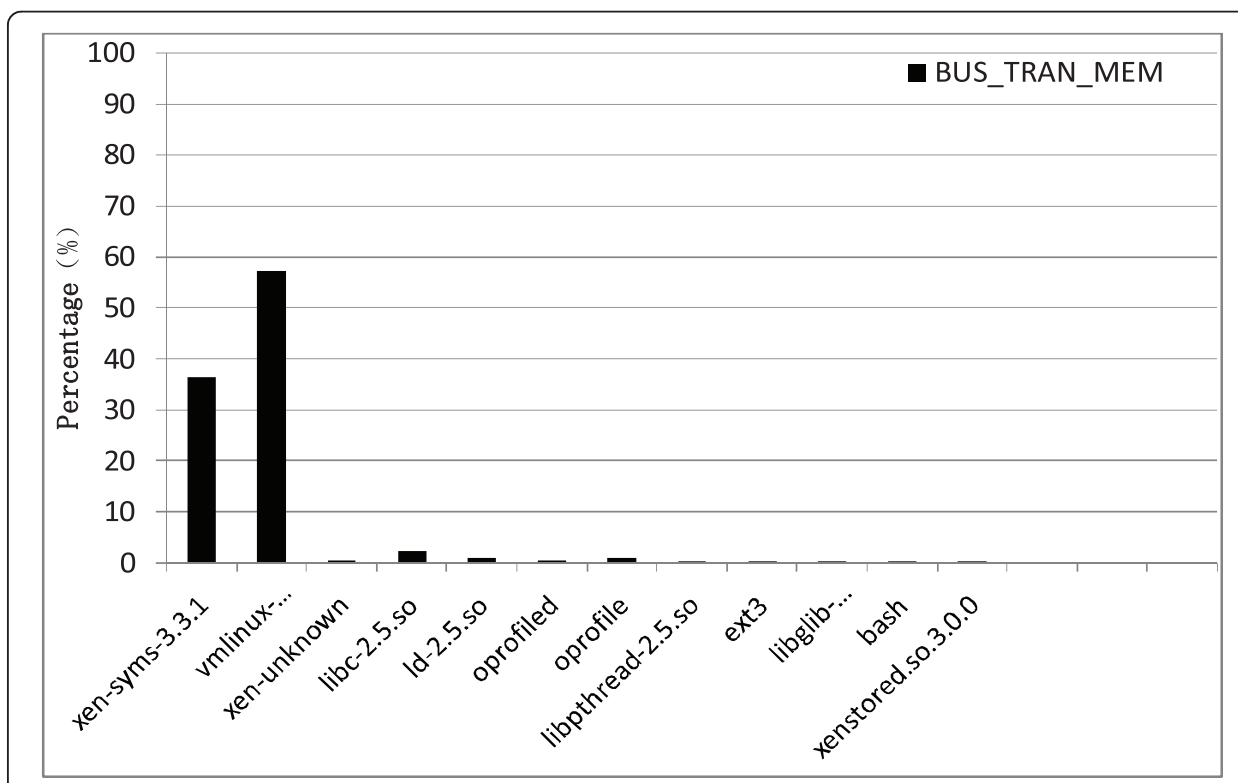

Figure 11 BUS_TRAN_MEM profiling in domain2.

From Figure 12 and 13 above, we can see top 5 hotspots of Gzip when CPU_CLK_UNHALTED and ITLB_MISS_RETIRED are monitored, and the performance data generated by Metis nearly match the result generated by Xenoprof.

\section{Conclusions}

In this paper, we present the design and implementation of a system-wide profiling toolkit: Metis. Comparing to all the existing profiling toolkits, Metis is different which

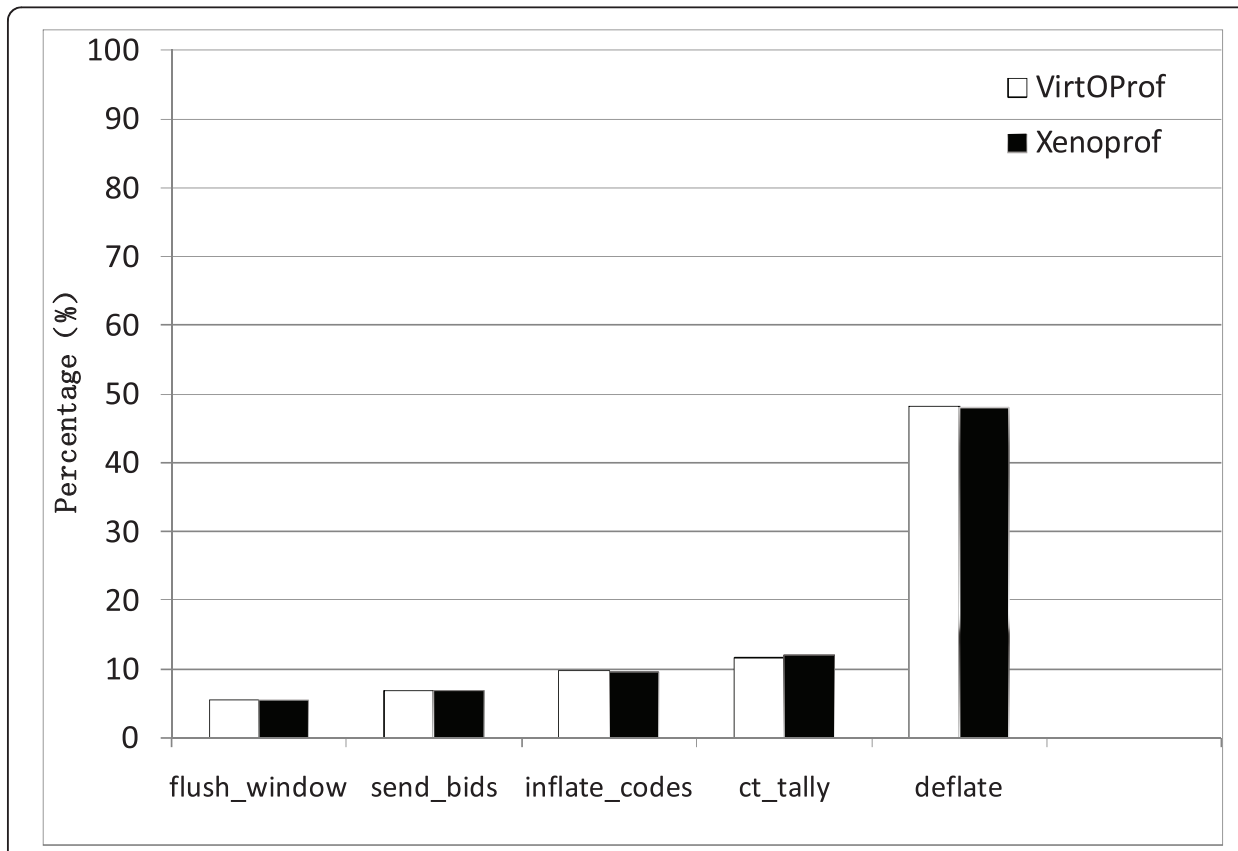

Figure 12 Comparison histogram of CPU_CLK_UNHALTED between VirtOprof and Xenoprof. 


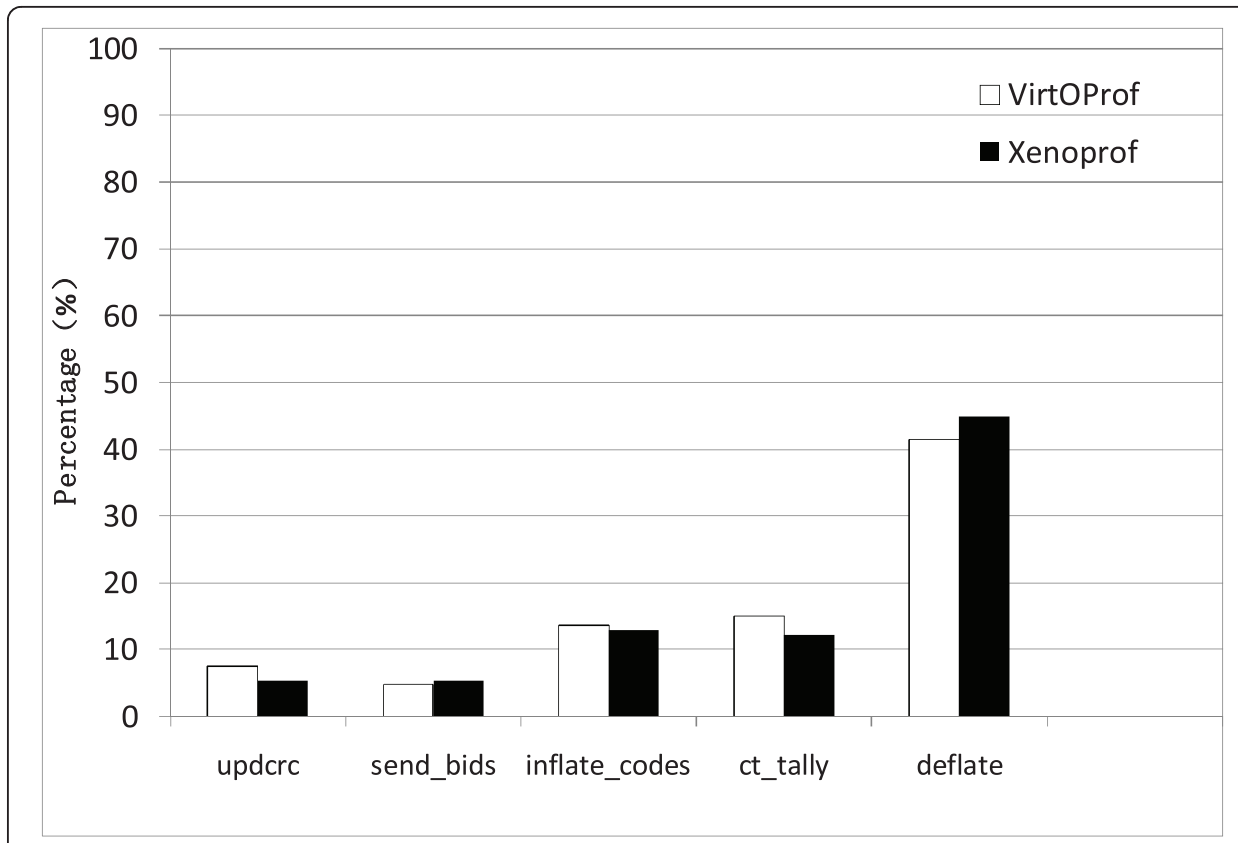

Figure 13 Comparison histogram of ITLB_MISS_RETIRED between VirtOprof and Xenoprof

enables multiple users in different virtual machines to monitor different CPU events simultaneously, and users in different domains can use this toolkit individually without affecting each other. To make Metis used individually, we choose to run background daemons in domains to profile for answering requests in time, and use the virtualization of hardware performance counters to satisfy our need. We modify Xen source code and Linux source code to support the virtualization of hardware performance counters. Besides this, a set of user level daemons are used too as the management unit in the toolkit, and we ensure the profiling accuracy in our implementation. We apply a popular benchmark to verify the correctness of Metis and its cool features.

\section{Acknowledgements}

This work is supported by National 973 Basic Research Program of China under grant No. 2007 CB310900 and National Natural Science Foundation under grant No.61003007.

\section{Author details}

${ }^{1}$ Services Computing Technology and System Lab, Cluster and Grid Computing Lab, School of Computer Science and Technology, Huazhong University of Science and Technology, Wuhan 430074, China ${ }^{2}$ School of Computer Science and Technology, Huazhong University of Science and Technology, Wuhan 430074, China

\section{Authors' contributions}

Xia Xie carried out the Metis architecture framework, participated in the message delivery mechanism design and drafted the manuscript. Haiou Jiang and Wenzhi Cao carried out performance evaluation. Pingpeng Yuan participated in analyze message delivery mechanism. Hai Jin and Tianruo Yang participated in its design and coordination and helped to draft the manuscript. All authors read and approved the final manuscript.

\section{Competing interests}

The authors declare that they have no competing interests.

Received: 1 November 2011 Accepted: 22 March 2012 Published: 22 March 2012

\section{References}

1. Padala P, Zhu X, Wang Z, Singhal S, Shin KG (2007) Performance evaluation of virtualization technologies for server consolidation. Technical Report HPL-2007-59R1, HP Laboratories

2. Barham P, Dragovic B, Fraser K, Harris SHT, Ho A, Neugebauer R, Pratt I, Warfield A (2003) Xen and the Art of Virtualization. the Nineteenth ACM Symposium on Operating Systems Principles (SOSP2003). New York, USA 
3. Gavrilovska A, Kumar S, Raj K, Gupta V, Nathuji R, Niranjan A, Saraiya P (2007) High-Performance Hypervisor Architectures: Virtualization in HPC Systems. 1st Workshop on System-level Virtualization for High Performance Computing, Canada

4. Huang W, Liu J, Abali B, Panda DK (2006) A Case for High Performance Computing with Virtual Machines. 20th ACM International Conference on Supercomputing (ICS (2006) Cairns. Queensland, Australia

5. Tikotekar A, Ong H, Alam S, Vallée G, Naughton T, Engelmann C, Scott SL (2009) Performance comparison of two virtual machine scenarios using an hpc application: a case study using molecular dynamics simulations. the $3 \mathrm{rd} \mathrm{ACM}$ Workshop on System-level Virtualization for High Performance Computing. USA, New York

6. Rosenblum M, Garfinkel T (2005) Virtual machine monitors: Current technology and future trends. IEEE Computer 38(5):39-47

7. Oprofile.http://oprofile.sourceforge.net. Accessed 10 Oct 2010

8. Menon A, Santos JR, Turner Y, Janakiraman GJ, Zwaenepoel W (2005) Diagnosing performance overheads in the Xen virtual machine environment. First International Conference on Virtual Execution Environments (VEE2005). Chicago, Illinois, USA

9. The VTune ${ }^{\text {TM }}$ Performance Analyzers.http://www.intel.com/software/products/vtune. Accessed: 20 Jan., 2011

10. VTune.http://en.wikipedia.org/wiki/TTune. Accessed: 10 Oct., 2011

11. Xenoprof.http://xenoprof.sourceforge.net. Accessed: 1 Feb., 2010

12. Santos JR, Turner $Y$, Janakiraman G, Pratt IA (2008) Bridging the gap between software and hardware techniques for $I / O$ Virtualization. ATC'08: USENIX (2008) Annual Technical Conference on Annual Technical Conference. USENIX Association, Berkeley, Korea

13. Tikotekar A, Vallée G, Naughton T, Ong HH, Engelmann C, Scott SL, Filippi AM (2008) Effects of virtualization on a scientific application running a hyperspectral radiative transfer code on virtual machines. the 2nd workshop on Systemlevel virtualization for high performance computing. ACM, New York, NY, USA

14. Tikotekar A, Vallée G, Naughton T, Ong H, Engelmann C, Scott SL (2008) An analysis of hpc benchmarks in virtual machine environments. 3 rd Workshop on Virtualization in High-Performance Cluster and Grid Computing. New York, USA

15. SPEC CPU2000.http://www.spec.org/cpu2000/. Accessed 10 Oct 2011

16. Hardware performance counters.http://en.wikipedia.org/wiki/Hardware_performance_counter. Accessed: 16 Apr., 2011

17. Configuration details.http://oprofile.sourceforge.net/doc/detailed-parameters.html. Accessed: 12 Aug., 2011

18. XenBus.http://wiki.xen.org/xenwiki/XenBus. Accessed: 10 Oct., 2011

19. Xenstore.http://wiki.xensource.com/xenwiki/XenStore. Accessed 10 Oct 2011

20. Cherkasova L, Gardner R (2005) Measuring CPU overhead for I/O processing in the xen virtual machine monitor. USENIX (2005) Annual Technical Confernce. USA, New York

doi:10.1186/2192-1962-2-8

Cite this article as: Xie et al:: Metis: a profiling toolkit based on the virtualization of hardware performance counters. Human-centric Computing and Information Sciences 2012 2:8.

\section{Submit your manuscript to a SpringerOpen ${ }^{\ominus}$ journal and benefit from:}

Convenient online submission

- Rigorous peer review

- Immediate publication on acceptance

- Open access: articles freely available online

- High visibility within the field

- Retaining the copyright to your article

Submit your next manuscript at $\gg$ springeropen.com 